\title{
Pancreatic islet cell tumour in a patient with familial adenomatous polyposis
}

\author{
C J R Stewart, C W Imrie, A K Foulis
}

\begin{abstract}
A case of familial adenomatous polyposis is reported in a 39 year old patient who underwent a Whipple's resection for adenoma of the duodenum. He subsequently developed a pancreatic glucagonoma. Endocrine neoplasia other than papillary carcinoma of thyroid has seldom been reported in association with FAP. The true incidence of islet cell tumours in FAP may be underestimated.
\end{abstract}

(F Clin Pathol 1994;47:860-861)

It has long been recognised that familial adenomatous polyposis (FAP) confers an autosomal dominant susceptibility to the development of multiple colonic neoplastic polyps and colonic carcinoma. More recently, and with appropriate management of large bowel disease, the extracolonic manifestations of FAP have assumed greater importance. ${ }^{12}$

Endocrine neoplasia other than papillary carcinoma of thyroid has seldom been reported in association with FAP. However, tumours of adrenal cortex and pituitary have been described, and a necropsy study by
Schneider et al suggested that the numbers of endocrine tumours are significantly increased in these patients. ${ }^{3}$ We report a case of a man with FAP who developed multiple adenomas of the duodenum, and was subsequently found to have a pancreatic glucagonoma.

\section{Case report}

A 39 year old man who had undergone total colectomy 20 years earlier for familial adenomatous polyposis presented with obstructive jaundice secondary to a large adenoma at the ampulla of Vater. Endoscopic sphincterectomy provided only temporary relief and, in view of the risk of carcinoma, the patient underwent a Whipple's resection. The excised duodenal specimen included multiple adenomas but none showed evidence of invasive malignancy. A few months later the patient presented with severe acute pancreatitis which was complicated by the development of a pancreatic pseudocyst and a splenic artery aneurysm. The aneurysm was resected together with the spleen and the tail of pancreas leaving a segment of body of pancreas. The patient remained well and non-diabetic three years after the last operation.
Department of Pathology, Glasgow Royal Infirmary C J R Stewart A K Foulis

Department of Surgery C W Imrie Correspondence to: Dr C J R Stewart, Department of Pathology, Glasgow Royal Infirmary, Glasgow G4 0SF

Accepted for publication 24 March 1994

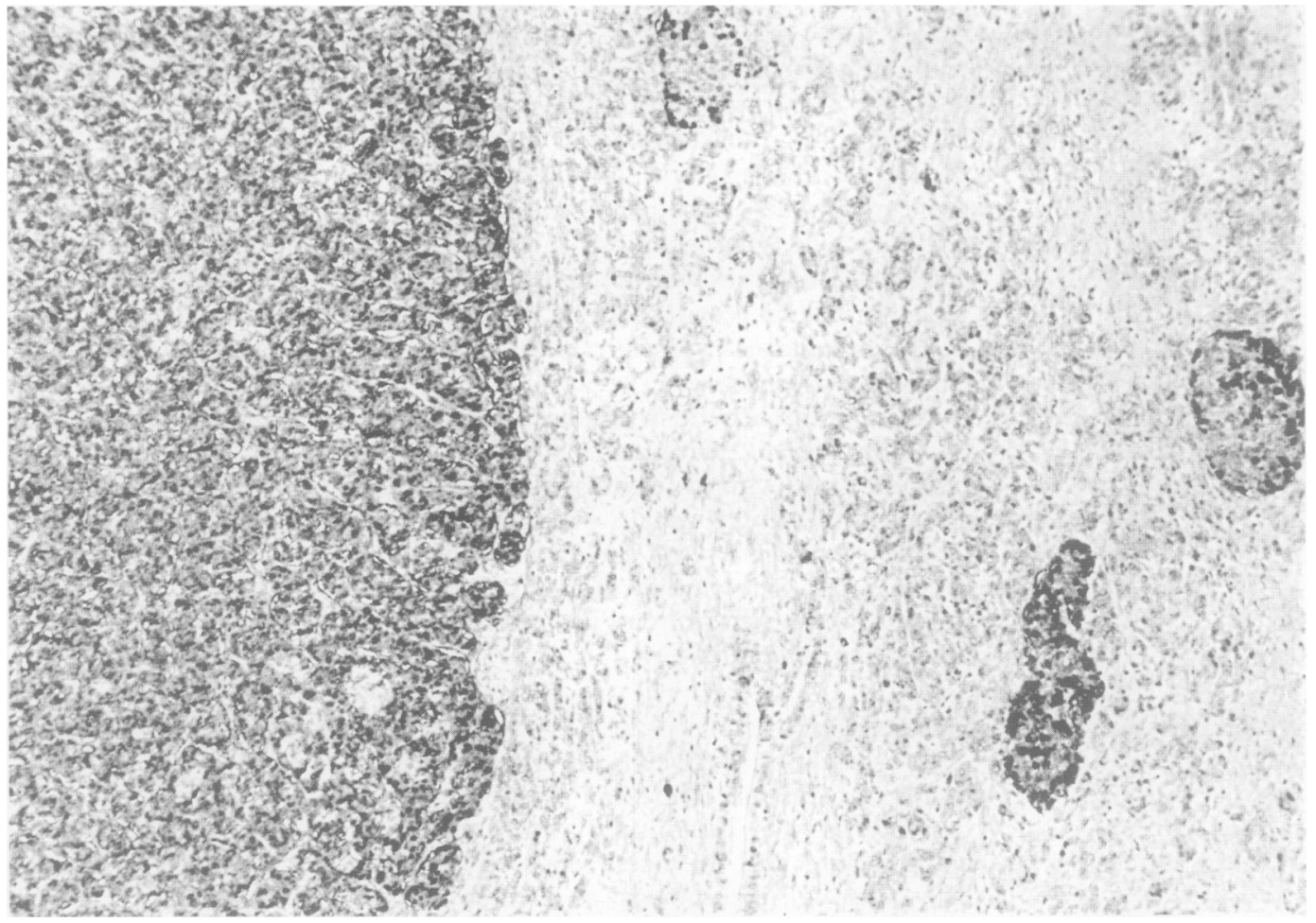

Islet cell tumour is strongly reactive with glucagon antiserum. Immunoreactive cells are also evident in adjacent islets (immunoperoxidase). 


\section{Pathological findings}

The specimen from the last procedure comprised a $200 \mathrm{~g}$ spleen with a $12 \times 8 \times 7.5$ $\mathrm{cm}$ encapsulated cystic lesion containing laminated thrombus at the hilum, and a $2.5 \times$ $1.5 \mathrm{~cm}$ segment of pancreatic tail. The latter was fibrotic and included on section a $1 \cdot 1 \times$ $0.8 \mathrm{~cm}$ circumscribed, focally haemorrhagic nodule close to one margin.

Histological analysis of the pancreatic nodule showed features typical of an islet cell tumour consisting of endocrine cells arranged in a trabecular or less commonly acinar pattern. Mild nuclear pleomorphism and occasional mitoses were identified. Immunocytochemistry demonstrated diffuse tumour cell reactivity for glucagon (figure) and focal staining with antisera to somatostatin and pancreatic polypeptide; there was no insulin immunoreactivity. Islets in the pancreatic parenchyma adjacent to the tumour gave the expected mixed pattern of hormone immunostaining. The parenchyma also showed features of obstructive pancreatitis. The cyst at the splenic hilum was a false aneurysm of the splenic artery which was considered secondary to pancreatitis associated arterial wall damage. The adjacent lymph nodes showed reactive changes while the splenic parenchyma was normal.

\section{Discussion}

Prevention of large bowel carcinoma in patients with FAP has resulted in increased awareness of the non-colonic manifestations of this condition. The risk of neoplasia in other gastrointestinal sites, particularly the peri-ampullary region, is well recognised ${ }^{4}$ and illustrated by the case presented here. Patients with FAP are also known to have an increased incidence of brain, bone, thyroid and adrenal tumours as well as desmoid disease, epidermal cysts, and abnormalities of retinal pigment epithelium. ${ }^{2}$ The spectrum of neoplasia in FAP continues to increase and recent reports have also documented associations with tumours of liver, ${ }^{5}$ gall bladder, ${ }^{6}$ bile duct, ${ }^{7}$ and uterus and ovary. ${ }^{8}$
To our knowledge, only one previous case of islet cell tumour in a polyposis syndrome has been reported (case 1 of the study by Schneider et al). ${ }^{3}$ This patient, a man of 58 , was found to have a "benign non-functioning" islet cell tumour at necropsy after developing metastatic sigmoid carcinoma. The apparent rarity of islet cell tumours in FAP may indicate that the association is coincidental. The history of pancreatitis in our patient may be relevant as islet cell tumours have been described in two patients with chronic pancreatitis. ${ }^{910}$ However, these cases differed in that the pancreatitis was of probable alcoholic aetiology and longer clinical duration. The true incidence of islet cell tumour in FAP may be underestimated as these tumours may be clinically asymptomatic, as in this case, and that previously described by Schneider and colleagues. ${ }^{3}$ Furthermore, the surgical prophylaxis of colonic carcinoma in affected patients may permit the development of further previously unrecognised conditions associated with FAP.

1 Parks TG. Extracolonic manifestations associated with familial adenomatous polyposis. Ann R Coll Surg Engl 1990;72:181-4.

2 Jagelman DG. Extra-colonic manifestations of familial adenomatous polyposis. Oncology 1991;5:23-7.

3 Schneider NR, Cubilla AL, Chaganti RSK. Association of endocrine neoplasia with multiple polyposis of the colon. Cancer 1983;51:1171-5.

4 Jagelman DG, DeCosse JJ, Bussey HJR. Upper gastrointestinal cancer in familial adenomatous polyposis. Lancet 1988;i:1149-51.

5 Laferla G, Kaye SB, Crean GP. Hepatocellular and gastric carcinoma associated with familial polyposis coli. $\mathcal{F}$ Surg Oncol 1988;38:19-21.

6 Bombi JA, Rives A, Astudillo E, Pera C, Cardesa A. Polyposis coli associated with adenocarcinoma of the gallbladder. Cancer 1984;53:2561-3.

7 Spigelman AD, Farmer KCR, James M, Richman PI, pigelman AD, Farmer KCR, James M, Richman PI,
Phillips RKS. Tumours of the liver, bile ducts, pancreas and duodenum in a single patient with familial adenomatous polyposis. Br f Surg 1991;78:979-80

8 Sohrabi AK, Nowzari P. Spectrum of clinical manifestations of familial adenomatous polyposis. $W V M e d f$ 1992;88:193-4.

9 Allison MC, Renfrew CC, Webb WJS, Chappell ME, Pounder RE. Neuroendocrine islet cell tumour producing gastrin and ACTH in a patient with calcifying chronic pancreatitis. Gut 1985;26:426-8.

10 Prescot RJ, Manson J, Haboubi NH. Malignant islet cell tumour arising in chronic pancreatitis. Histopathology 1993;22:499-501. 